$3(R=0.597, p<0.001, n=38 ; R=0.462, p=0.009$, $\mathrm{n}=31)$. TNF-alpha and IL-10 both correlated with free TF in cord samples $(R=0.536, p=0.012, n=31$, and $R=0.610, p=0.003, n=22$ ). No correlations were found between cytokines and F1+2.

Conclusions: Both pro- and anti-inflammatory cytokines correlated with high TF concentration. A difference seems to exist between in utero (cord) and postnatal TF regulation: in cord plasma TNFalpha as well as IL-10 showed strong associations with TF but lost this role to IL-8 postnatally. Free TF failed to contribute to the rate of thrombin generation. This shift of TF from coagulation to inflammation may have effects on pathogenesis of lung injury, as well as on extrapulmonary morbidity in preterm infants.

94

\section{ROUTINE COAGULATION SCREENING IN NICU- TO DO OR NOT TO DO? REVIEW OF 2 U.K TERTIARY NEONATAL UNITS}

P. Muthukumar ${ }^{1}$, K. Catford ${ }^{1}$, V. Venkatesh ${ }^{1}$, S. Reddy ${ }^{1}$, A. Curley ${ }^{1}$, P. Clarke ${ }^{2}$

${ }^{1}$ Neonatal Unit, Cambridge University Hospital NHS Foundation Trust, Cambridge, ${ }^{2}$ Neonatal Unit, Norfolk \& Norwich University Hospitals NHS Trust, Norwich, UK

Introduction: Routine coagulation screening of neonates admitted for intensive care is common. There is little evidence of efficacy of fresh frozen plasma (FFP) in the treatment of isolated abnormal coagulation values.

Objective: To compare transfusion rates of FFP between 2 tertiary neonatal units with similar admission rates one using routine (NICU1) and other targeted coagulopathy screening (NICU2). Secondary objective was to assess the proportions of cases treated in each unit with FFP whose coagulation parameters reverted to the normal range.

Methods: Retrospective review of neonates receiving FFP from Jan 2003- March 2008 in two UK tertiary-level neonatal units

Results: 164 neonates received 268 FFP transfusions. $131 / 164(80 \%)$ infants in NiCU1 compared with $33 / 164(20 \%)$ in NICU2 received FFP. Predominant reason for transfusion was abnormal laboratory measures of coagulation $(77 \%$ in $\mathrm{NICU} 1$ and $47 \%$ in NICU 2). Active bleeding only accounted for $15 \%$ of transfusions. Pre and post transfusion coagulation studies (within 24 hours) were measured in $72 \%$. In only $15 \%$ did administration of FFP corrected laboratory parameters to the normal range for gestational age, in more than half of instances this occurred with diagnosis of isolated abnormal coagulation values. There was no significant difference in the proportion of babies with corrected values post FFP between two units.

Conclusion: Routine coagulopathy screening may increase the number of FFP transfusions. FFP administration did not correct laboratory parameters in majority of transfusions. The risks of transfusions should be balanced against limited clinical evidence for its administration in isolated coagulopathy.

95

\section{HIGH COPEPTIN (ARGININE-VASOPRESSIN) CONCENTRATIONS IN UMBILICAL CORD BLOOD AFTER VAGINAL DELIVERY AND BIRTH ACIDOSIS}

S. Wellmann ${ }^{1}$, J. Benzing ${ }^{2}$, G. Cippa ${ }^{1}$, D. Admaty ${ }^{1}$, R. Creutzfeldt ${ }^{1}$, E. Beinder ${ }^{3}$, G. Szinnai ${ }^{4}$, O. Lapaire ${ }^{5}$, N.G. Morgenthaler ${ }^{6}$, U. Haagen ${ }^{6}$, C. Bührer ${ }^{7}$, H.U. Bucher ${ }^{1}$

${ }^{1}$ Department of Neonatology, University Hospital Zürich, Zürich, ${ }^{2}$ Department of Neonatology, University Children's Hospital Basel, Basel, ${ }^{3}$ Department of Obstetrics, University Hospital Zürich, Zürich, ${ }^{4}$ Pediatric Endocrinology, University Children's Hospital Basel, ${ }^{5}$ Devision of Obstetrics and Gynecology, University Hospital Basel, Basel, Switzerland, ${ }^{6}$ Research Department, B.R.A.H.M.S.

AG, Hennigsdorf, 'Department of Neonatology,

Charité University Medical Center, Berlin, Germany

Background: The pituitary-secreted nonapeptide Arginine-Vasopressin (AVP) is unstable and therefore unsuited for diagnostic use but its secretion can be gauged by measuring copeptin, the C-terminal portion of the AVP precursor (CT-proAVP). We determined copeptin in umbilical cord blood and at 3 days of age in order to establish normal values and perinatal factors affecting copeptin concentrations.

Methods: Paired arterial/venous umbilical cord samples were obtained from 117 infants and umbilical venous-only samples from additional 46 infants. In 102 infants, blood was also obtained at 3 days of life. Copeptin levels were determined using the CT-proAVP-Luminescence-immunoassay. 